\title{
Promoting the Introduction of Lifelong Learning Related Concepts in the Description of Information Resources Using Metadata Technology
}

\author{
Rocío García Robles \\ Institute for Prospective Technological Studies \\ rocio.garcia@irc.es
}

\begin{abstract}
The rise of Internet has created an urgent need to define widely agreed-upon methods and vocabularies for describing its contents in a consistent and orderly manner. This necessity can be satisfied by means of the use of Metadata Schemas. In achieving this aim in relation to the Educational, Vocational and Training sector, several initiatives have emerged at an international level, but there is a need for integrating core skills and competencies issues, which are very important in relation to the new paradigm of Lifelong Learning $(L L L)$ and especially regarding with informal education and training, into the metadata solutions that are arisen in the framework of those initiatives.
\end{abstract}

\section{Introduction}

In order to improve the retrieval of information from electronic data sources, since a few years ago a number of Metadata initiatives have arisen, developing metadata schemas and specifications to describe all kind of information sources. But how can Metadata be defined?. Metadata is a broad term that covers many types of "structured data about data" - and it can be applied to any kind of information resource from traditional ones such as library catalogues to new forms of technical and descriptive data for Web resources. Therefore Metadata is used to describe an information resource, but Metadata can serve a variety of purposes, not only for localisation aims identifying a resource that meets a particular information need, but also evaluating their suitability for use or tracking the characteristics of resources for maintenance or usage over time.

In order to allow a common platform for applying Metadata Schemas ensuring interoperability between all information resources, it is important to get consensus among all related initiatives. This process culminates with the arising of worldwide standards on metadata schemas, as in the development of global specifications to be used freely by any person, company or institution. An area where Metadata have been successfully agreed-upon is on the Vocational, Education and Training (VET) sector.

According to one of the Metadata Watch Reports delivered by the SCHEMAS Project ${ }^{[1]}$, there are four important Metadata Initiatives on the Education and Training domain:

1. First of these initiatives in the IEEE LTSC LOM ${ }^{[2]}$, The IEEE (The Institute of Electrical and Electronics Engineers, Inc) LTSC (Learning Technology Standardization Committee)

2. The second initiative is the $\mathrm{DCMI}^{[3]}$ : (Dublin Core Metadata Initiative)

3. The third initiative is the CEN (European Committee for Standardization) ISSS (Information Society Standardization System) LTWS ${ }^{[4]}$ (Learning Technology Workshops).

4. The fourth initiative is the ISO (ISO: International Standardization Office) IEC JTCl ${ }^{[5]}$ (Joint Technical Committee) SC36 (SubCommittee number 36).

\section{Rationale}

In an innovation-driven economy, and a world characterised by the emergence of a mosaic of lifestyles and intermixing of cultures, a commitment to continuous learning and the generation of new knowledge has become vital to sustain economic, social and cultural development. This applies equally to individuals, organisations, whole communities, regions and countries in a worldwide scope ${ }^{[6]}$.

Therefore, there is a new paradigm on the Vocational Education and Training (VET) field, which is called "Lifelong Learning". This term describes a goal set by the most developed countries, whereby all citizens would participate in learning activities over the complete course of their lives. This includes structured learning ranging from initial formal education, to job related training courses provided by employers, public labour market training schemes, and adult education programmes. But it also includes the informal learning which takes place in all varieties of life-situations, outside educational institutions and structured learning activities, such as early childhood learning, learning-by-doing, selfmotivated study and learning, learning via social interaction, learning from the mass-media, and so on. In this sense, the term Life-Wide Learning is sometimes used to describe the breadth and variety of formal and informal learning that takes place in each life phase, and 
includes an implicit message that the informal aspects be left less to chance than before ${ }^{[6]}$.

But Lifelong learning is still far from a reality and must continue to be pursued vigorously to provide all groups in society with proper learning opportunities, regardless of age, gender, ethnicity and basic formal qualifications $^{[6]}$.

In both cases, formal and informal learning, (core) skills $^{[7]}$ and competencies are key issues that must be tackled as "drivers" for learning ${ }^{[6]}$. They are essential components of modern VET systems, becoming important means by which innovative teaching and learning practices are implemented into educational institutions, leading to an stronger emphasis on learning by doing (which is especially important in relation to informal learning $)^{[8]}$.

Although main Metadata initiatives on the VET sector are supposed to be aware of the new paradigm of Lifelong Learning, there seems to be a gap in introducing those key issues, skills and competencies, into the current approved versions of Metadata Schemas. But, as a previous stage for integrating skills and competency concepts into Metadata Schemas and Specifications, there is a need to get agreement on the definition of all these related concepts and also on the possible ways of categorization $^{[10]}$.

\section{Conclussion: Advantages of considering Core Skills discourse in relation to Metadata Initiatives}

As it was previously explained, skills are the "drivers" for formal and especially informal learning in the framework of LLL. In this sense, and in relation to metadata initiatives, it is true that there are some national approaches to produce taxonomies on competencies, (NVQs), but there seems to be little concern, if any, in trying to introduce all these important concepts into metadata schemas which will be used to describe information resources on VET.

Main advantages of integrating skills and competencies discourse in relation to the Metadata Initiatives would be: to offer a new way for localisation of information, up-to-the-minute curricula development and connection between VET and the labour market.

Therefore, core skills can guide the development of new up-to-date curricula, because to include skills information into metadata schemas would serve to give a tool to different targeted user groups (teacher, learner,) to be able to choose learning materials (for example, a course) using as searching criteria the type of skills the user group wants to acquire, and not only focusing the search on content details of the learning material. This is particularly relevant to informal learning, where skills are the "drivers" for learning activities.

Moreover, as it was mentioned above, the ideal aim would be to be able to specify skills with the same terminology used in the labour market in relation, e.g., to the profile of the employees who are already working or who are demanded in the workplace. Therefore, to include skills information as part of the metadata structure could improve the process of searching for information using the terms of reference of the labour market.

After the previous analysis we can conclude that metadata schemas that, according to the original definition, are used to describe information resources, should be linked to core skills and competencies concepts.

\section{Notes \& References}

${ }^{[1]}$ Document Number: SCHEMAS-PwC-WP2-D22-Final20000602. http://www.schemas-forum.org/

${ }^{[2]}$ IEEE LTSC Initiative: http://ltsc.jeee.org/

${ }^{[3]}$ Dublin Core Metadata Initiative (DCMI): http://purl.org/DC/

${ }^{[4]}$ CÉN/ISSS LTWS Initiative: http://www.cenorm.be/isss/

${ }^{[5]}$ ISO/IEC JTC1 SC36: http://itc/ sc 36.org/

${ }^{[6]}$ The IPTS Futures Project Report Series N. 14: "Knowledge and Learning: Towards a Learning Europe"

[7] For the purposes of this study, the terms skills and competencies are considered equivalent. According to Neville Bennett's definition (Bennett N., Dunne E., Carré C., (1999), "Patterns of Core and Generic Skill Provision in Higher Education", Higher Education 37), the word "core" refers to disciplinary skills, that are generic enough to represent the skills which can support study in any discipline, and which can potentially transferred to a range of contexts, in formal and informal education or in the workplace. There are many ways of classifying skills. Those skills go from skills for learning and developing competence to skills for looking for opportunities to start or improve businesses and, generally speaking, skills for developing effective performance across a wide range of settings at work but also in social and economic life.

${ }^{181}$ Report on "Development of Core Skills Training in the Partner Countries", June 1998, European Training Foundation (ETF). http://www.etf.eu.int/etfweb.nsf/pages/downloadthemel

${ }^{[10]}$ García Robles, R. April 2001, "Metadata on Education and Training promises Lifelong Learning", IPTS Report. 